
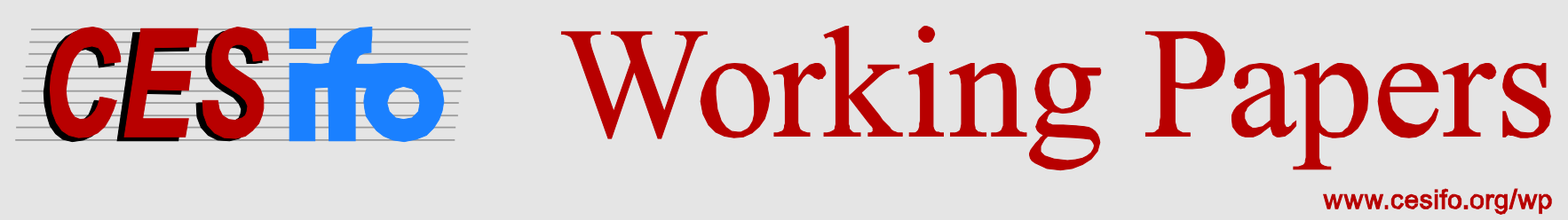

\title{
Signs of Impact Effects in Time Series Regression Models
}

\author{
M. Hashem Pesaran \\ Ron P. Smith
}

\author{
CESIFO WORKING PAPER NO. 4433 \\ CATEGORY 12: EMPIRICAL AND THEORETICAL METHODS \\ OCTOBER 2013
}

An electronic version of the paper may be downloaded

- from the SSRN website:

- from the RePEc website:

- from the CESifo website:

WwW.SSRN.com

Www.RePEc.org

www.CESifo-group.org/wp

\section{CESifo}




\title{
Signs of Impact Effects in Time Series Regression Models
}

\begin{abstract}
In this paper we consider the problem of interpreting the signs of the estimated coefficients in multivariate time series regressions where the regressors are correlated. Using a continuous time model, we argue that focussing on the signs of individual coefficients in such regressions could be misleading and argue in favour of allowing for the indirect effects that arise due to the historical correlations amongst the regressors. For estimation from discrete time data we show that the sign of the total impact, including the direct and indirect effects, of a regressor can be obtained using a simple regression that only includes the regressor of interest.
\end{abstract}

JEL-Code: C100, C500.

Keywords: regression coefficients, impact effects.

\author{
M. Hashem Pesaran \\ Department of Economics \\ University of Southern California \\ Los Angeles / California / USA \\ pesaran@usc.edu
}

\author{
Ron P. Smith \\ Department of Economics, Mathematics \\ \& Statistics \\ Birkbeck, University of London \\ London / United Kingdom \\ r.smith@bbk.ac.uk
}

October 7, 2013

We are grateful to Alex Chudik and Stephen Wright for comments. 


\section{Introduction}

In designing an experiment to measure the effect of some input variables, say $x_{j}, j=1,2, . ., k$, on an outcome variable, say $y$, one tries to ensure that the individual inputs are orthogonal and have maximal variance. In contrast the observational time series data used in economics are typically realisations from highly correlated continuous stochastic processes not subject to such experimental control. There is thus an issue of how to measure the effect of a particular variable, say $x_{i}$, and determine the sign of that effect. The standard procedure in a multiple linear regression context is to use the estimated regression coefficient of $x_{i}$. This measures the effect of a hypothetical change in $x_{i}$, holding $x_{j}, j \neq i$, constant, as if we were in fact able to conduct an experiment and make the inputs orthogonal. But in almost all economic applications we are not able to control the inputs and to use the same language in the two different settings can be misleading. We argue that in time series analysis rather than focussing on the signs of individual coefficients in such multiple regressions, holding the other variables constant, we should measure a total impact effect which allows for the indirect induced changes that arise due to the historical correlations amongst the regressors. The limitation of the usual ceteris paribus approach (which relies on assuming other variables are held constant) lies in the fact that it ignores the stochastic inter-dependence of the regressors which we need to allow for in time series economic applications. ${ }^{1}$ Similar issues arise in the derivation of impulse response functions for the analysis of dynamic models and have been discussed by Koop, Pesaran and Potter (1996) and Pesaran and Shin (1998). We follow a similar line of reasoning below, but focus on impact effects rather than responses over time.

The effects we are interested in are usually characterised as derivatives and to clarify the counterfactuals that we are considering, we first examine what we mean by the effect of a variable in a continuous time model, which allows us to be precise about the nature of the derivatives of the underlying stochastic processes that enter a multivariate regression. ${ }^{2}$ We then consider estimation, which uses discrete data, using the linear case as an example.

\section{A stochastic regression model in continuous time}

Suppose that the outcome variable, $y(t)$, and the $k$ regressors, $x_{j}(t)$ are stochastic processes observed over continuous time, $t$, and we are interested in identifying the effects of a small change in the focus regressor, $x_{i}(t)$, on $y(t)$. Assume that

$$
y(t)=F\left(x_{1}(t), x_{2}(t), \ldots, x_{k}(t)\right)+u(t),
$$

\footnotetext{
${ }^{1}$ In Latin we want the effect to be mutatis mutandis rather than ceteris paribus.

${ }^{2}$ In pure cross section regressions where the regressors are randomized and/or matched across units, the issues raised in this paper might not arise. Here our focus is on time series regressions.
} 
where $F($.$) is twice differentiable function and \mathbf{x}(t)=\left(x_{1}(t), x_{2}(t), \ldots, x_{k}(t)\right)^{\prime}$ is a realization of a stochastic process. To simplify the discussion, further suppose that $\mathbf{x}(t)$ follows the multivariate drift-diffusion process

$$
d \mathbf{x}(t)=\boldsymbol{\mu}_{t} d t+\boldsymbol{\Omega}_{t}^{1 / 2} d \mathbf{B}(t),
$$

where $\boldsymbol{\mu}_{t}$ and $\boldsymbol{\Omega}_{t}$ are the mean and the covariance matrix of $d \mathbf{x}(t)$, respectively, and $\mathbf{B}(t)$ is a $k \times 1$ vector of Brownian process, assumed to be distributed independently of $u(t)$, where $d x_{i}(t)=x_{i}(t+d t)-x_{i}(t), d u(t)=u(t+d t)-u(t)$, and $d t$ represents a small time increment such that $(d t)^{2}$ and its higher powers are negligible. Applying Ito's lemma to $y(t)$ gives

$$
d y(t)=\sum_{j=1}^{k} \frac{\partial F(\mathbf{x}(t))}{\partial x_{j}(t)} d x_{j}(t)+\frac{1}{2} \sum_{r=1}^{k} \sum_{s=1}^{k} \frac{\partial^{2} F(\mathbf{x}(t))}{\partial x_{r}(t) \partial x_{s}(t)} d x_{r}(t) d x_{s}(t)+d u(t) .
$$

This is a stochastic differential equation that maps the changes in all $k$ regressors on the outcome variable. Thus, the effect of a change in $x_{i}(t)$ on $y(t)$ is not confined to its direct effect, but also involves the indirect effects through the correlation of $d x_{i}(t)$ with the other regressors, represented in (2). Also,given the stochastic nature of the processes, all the increments $d y(t), d x_{1}(t), \ldots, d x_{k}(t)$, and $d u(t)$ are random draws and cannot be set to zero, even if the regressors are orthogonal. In this stochastic setting it is more appropriate to consider mean changes to $y(t)$ as a result of mean incremental shifts in $x_{i}(t)$. Accordingly, we propose to measure the effect on $y(t)$ as a result of an incremental change to $x_{i}(t)$ by

$$
\begin{aligned}
E\left[d y(t) \mid d x_{i}(t)\right]= & \sum_{j=1}^{k} \frac{\partial F(\mathbf{x}(t))}{\partial x_{j}(t)} E\left[d x_{j}(t) \mid d x_{i}(t)\right] \\
& +\frac{1}{2} \sum_{r=1}^{k} \sum_{s=1}^{k} \frac{\partial^{2} F(\mathbf{x}(t))}{\partial x_{r}(t) \partial x_{s}(t)} E\left[d x_{r}(t) d x_{s}(t) \mid d x_{i}(t)\right] \\
& +E\left[d u(t) \mid d x_{i}(t)\right],
\end{aligned}
$$

where $E\left[. \mid d x_{i}(t)\right]$ denotes the conditional expectations operator. Given that $\mathbf{x}(t)$ is known at time $t$, the partial derivatives are also known and can be taken outside the expectations operator. In the context of regression models it is typically assumed that the regressors are exogenous so $E\left[d u(t) \mid d x_{i}(t)\right]=0$.

The above expression should be compared to the partial derivative of $F($. with respect to $x_{i}(t)$, namely $\partial F(\mathbf{x}(t)) / \partial x_{i}(t)$, used in the literature to measure the effects of $x_{i}(t)$ on $y(t)$. The two expressions, $E\left[d y(t) \mid d x_{i}(t)\right]$ and $\partial F(\mathbf{x}(t)) / \partial x_{i}(t)$, coincide only under very restrictive assumptions typically not satisfied in economic applications.

\subsection{Some examples}

In the specific case of the linear model, (1) is:

$$
y(t)=\beta_{0}+\beta_{1} x_{1}(t)+\beta_{2} x_{2}(t)+\ldots+\beta_{k} x_{k}(t)+u(t),
$$


(3) is:

$$
d y(t)=\sum_{j=1}^{k} \beta_{j} d x_{j}(t)+d u(t) .
$$

Then taking expectations (4) is:

$$
E\left[d y(t) \mid d x_{i}(t)\right]=\sum_{j=1}^{k} \beta_{j} E\left[d x_{j}(t) \mid d x_{i}(t)\right]+E\left[d u(t) \mid d x_{i}(t)\right] .
$$

Again assuming exogeneity $E\left[d u(t) \mid d x_{i}(t)\right]=0$, and writing the conditional expectations of the other regressors as:

$$
E\left[d x_{j}(t) \mid d x_{i}(t)\right]=\gamma_{j i}(t) d x_{i}(t), i \neq j,
$$

where $\gamma_{j i}(t)$ can be derived from the parameters of (2), the total impact effect on $y(t)$ of an incremental change in $x_{i}(t)$ can be written

$$
E\left[d y(t) \mid d x_{i}(t)\right]=\lambda_{i}(t) d x_{i}(t)
$$

where

$$
\lambda_{i}(t)=\sum_{j=1}^{k} \beta_{j} \gamma_{j i}(t), \text { with } \gamma_{i i}(t)=1,
$$

which may have a different sign from $\beta_{i}$.

As a second example suppose that we have two variables and an interaction effect so that (1) is:

$$
y(t)=\beta_{0}+\beta_{1} x_{1}(t)+\beta_{2} x_{2}(t)+\beta_{12} x_{1}(t) x_{2}(t)+u(t),
$$

then (3) is

$$
\begin{aligned}
d y(t)= & {\left[\beta_{1}+\beta_{12} x_{2}(t)\right] d x_{1}(t)+\left[\beta_{2}+\beta_{12} x_{1}(t)\right] d x_{2}(t) } \\
& +\beta_{12} d x_{1}(t) d x_{2}(t)+d u(t)
\end{aligned}
$$

Then taking expectations conditional on, $d x_{1}(t)$, a given small incremental change in $x_{1}(t)$, and for given values of $x_{1}(t)$ and $x_{2}(t)$ we have:

$$
\begin{aligned}
E\left[d y(t) \mid d x_{1}(t)\right]= & {\left[\beta_{1}+\beta_{12} x_{2}(t)\right] d x_{1}(t)+\left[\beta_{2}+\beta_{12} x_{1}(t)\right] E\left(d x_{2}(t) \mid d x_{1}(t)\right] } \\
& +\beta_{12} E\left(d x_{1}(t) d x_{2}(t) \mid d x_{1}(t)\right)+E\left(d u(t) \mid d x_{1}(t)\right] .
\end{aligned}
$$

Once again letting $E\left[d x_{2}(t) \mid d x_{1}(t)\right]=\gamma_{21}(t) d x_{1}(t)$, then with exogenous regressors we obtain

$$
\begin{aligned}
E\left[d y(t) \mid d x_{1}(t)\right]= & \left\{\left[\beta_{1}+\beta_{12} x_{2}(t)\right]+\left[\beta_{2}+\beta_{12} x_{1}(t)\right] \gamma_{21}(t)\right\} d x_{1}(t) \\
& +\beta_{12} \gamma_{21}(t)\left[d x_{1}(t)\right]^{2}
\end{aligned}
$$


and since for small increments $\left[d x_{1}(t)\right]^{2}$ is negligible relative to the earlier terms, the impact effect of a small change in $x_{1}(t)$ on $y(t)$ is given by

$$
\lambda_{1}(t)=\left[\beta_{1}+\beta_{12} x_{2}(t)\right]+\left[\beta_{2}+\beta_{12} x_{1}(t)\right] \gamma_{21}(t) .
$$

As a third example, suppose that we have a quadratic function of a single regressor, so that (1) is:

$$
y(t)=\beta_{0}+\beta_{1} x(t)+\beta_{2}[x(t)]^{2}+u(t) .
$$

Clearly here it does not make any sense to ask what is the effect on $y(t)$ of a change in $x(t)$, holding $x(t)^{2}$ fixed. Then (3) takes the form

$$
d y(t)=\left[\beta_{1}+2 \beta_{2} x(t)\right] d x(t)+\beta_{2}(d x(t))^{2}+d u(t),
$$

and since for small increments $[d x(t)]^{2}$ is negligible, we have

$$
E[d y(t) \mid d x(t)]=\left[\beta_{1}+2 \beta_{2} x(t)\right] d x(t),
$$

which is the standard result.

\section{Estimation of impact effects in the linear case}

Consider now the problem of estimating $\lambda_{i}(t)$ using observations $y_{t}, x_{j t}$, for $j=1,2, \ldots, k$; obtained at discrete time (intervals) points $t=1,2, \ldots, T$. The discrete-time regression model corresponding to (5) is given by

$$
y_{t}=\beta_{0}+\sum_{j=1}^{k} \beta_{j} x_{j t}+u_{t},
$$

for $t=1,2, \ldots, T$ where we assume that the classical assumptions hold, namely $\beta_{i}$ are fixed constants, $u_{t} \backsim i i d\left(0, \sigma^{2}\right)$, and $E\left(u_{t} \mid x_{j t}\right)=0$ for all $j$ and $t$. We shall also assume that the relationships between the regressors are linear, such that $\gamma_{j i}$ are fixed constants. The analysis can be readily extended to simultaneous equation systems and dynamic models. ${ }^{3}$ To estimate the overall (direct and indirect) impact effect of $x_{i t}$ on $y_{t}$, as given by $\lambda_{i}$ in (10), we first note that in the context of the linear regression model, (17), we have

$$
\begin{aligned}
E\left(y_{t} \mid x_{i t}\right) & =\beta_{0}+\sum_{j=1}^{k} \beta_{j} E\left(x_{j t} \mid x_{i t}\right) \\
& =\beta_{0}+\boldsymbol{\beta}^{\prime} E\left(\mathbf{x}_{t} \mid x_{i t}\right) .
\end{aligned}
$$

\footnotetext{
${ }^{3}$ There is a large literature devoted to deriving the exact discrete model satisfied by observations generated by a continuous time model, e.g. Bergstrom (1984).
} 
To model the interdependence of the regressors and obtain estimates for $\gamma_{j i}$ in (8), we adopt the linear approximation for $E\left(\mathbf{x}_{t} \mid x_{i t}\right)$ which we write $a^{4}$

$$
E\left(\mathbf{x}_{t} \mid x_{i t}\right)=\boldsymbol{\mu}+\frac{\operatorname{Cov}\left(\mathbf{x}_{t}, x_{i t}\right)}{\sigma_{i i}}\left(x_{i t}-\mu_{i}\right),
$$

where

$$
\operatorname{Cov}\left(\mathbf{x}_{t}, x_{i t}\right)=\left(\begin{array}{c}
\sigma_{1 i} \\
\sigma_{2 i} \\
\vdots \\
\sigma_{k i}
\end{array}\right)=\boldsymbol{\sigma}_{i}
$$

Using these results in (18) we have

$$
E\left(y_{t} \mid x_{i t}\right)=\beta_{0}+\boldsymbol{\beta}^{\prime}\left(\boldsymbol{\mu}-\sigma_{i i}^{-1} \boldsymbol{\sigma}_{i} \mu_{i}\right)+\sigma_{i i}^{-1} \boldsymbol{\beta}^{\prime} \boldsymbol{\sigma}_{i} x_{i t} .
$$

Therefore, the overall impact effect of $x_{i t}$ on $y_{t}$, corresponding to (10) is also given by (using $\gamma_{j i}=\sigma_{j i} / \sigma_{i i}$ )

$$
\lambda_{i}=\sigma_{i i}^{-1} \boldsymbol{\beta}^{\prime} \boldsymbol{\sigma}_{i}=\sum_{j=1}^{k} \beta_{j} \gamma_{j i} .
$$

Notice that this simplifies to $\beta_{i}$ only when $x_{i t}$ is orthogonal to all the other regressors.

This analysis should be regarded as an approximate solution since, as assumed above in the continuous time case, the covariance matrix of the regressors may vary over time. But for the purpose of checking the sign of the impact effects against our a priori knowledge of them this may not be a serious problem.

To estimate the overall impact effect, $\lambda_{i}=\sigma_{i i}^{-1} \boldsymbol{\beta}^{\prime} \boldsymbol{\sigma}_{i}$, we first note that

$$
\boldsymbol{\sigma}_{i}=\mathbf{\Sigma} \mathbf{s}_{i}
$$

where $\mathbf{s}_{i}$ is a $k \times 1$ selection vector with all elements equal to zero except for its $i$-th element which is set equal to unity. Consistent estimates of the elements of $\Sigma$ are given by

$$
\hat{\sigma}_{i j}=\frac{\sum_{t=1}^{T}\left(x_{i t}-\bar{x}_{i}\right)\left(x_{j t}-\bar{x}_{j}\right)}{T-1},
$$

where $\bar{x}_{i}$ is the sample mean of $x_{i t}$. In matrix notation

$$
\hat{\mathbf{\Sigma}}=\frac{\mathbf{X}^{\prime} \mathbf{M X}}{T-1}
$$

where $\mathbf{X}$ is the $T \times k$ matrix of observations on $\left(x_{j t}, j=1,2, \ldots, k\right)$, and $\mathbf{M}=\mathbf{I}_{T}-$ $\boldsymbol{\iota}\left(\boldsymbol{\iota}^{\prime} \boldsymbol{\iota}\right)^{-1} \boldsymbol{\iota}^{\prime}$, with $\boldsymbol{\iota}^{\prime}=(1,1, \ldots, 1)^{\prime}$. The OLS estimator of $\boldsymbol{\beta}$ is given by

$$
\hat{\boldsymbol{\beta}}=\left(\mathbf{X}^{\prime} \mathbf{M X}\right)^{-1} \mathbf{X}^{\prime} \mathbf{M y}
$$

\footnotetext{
${ }^{4}$ This expression is exact if the distribution of $\mathbf{x}_{t}$ is multi-variate normal.
} 
where $\mathbf{y}=\left(y_{1}, y_{2}, \ldots, y_{T}\right)^{\prime}$. Hence

$$
\begin{aligned}
\hat{\lambda}_{i} & =\hat{\sigma}_{i i}^{-1} \hat{\boldsymbol{\beta}}^{\prime} \hat{\boldsymbol{\sigma}}_{i}=\hat{\sigma}_{i i}^{-1} \mathbf{y}^{\prime} \mathbf{M X}\left(\mathbf{X}^{\prime} \mathbf{M X}\right)^{-1}\left(\frac{\mathbf{X}^{\prime} \mathbf{M X}}{T-1}\right) \mathbf{s}_{i} \\
& =\frac{\mathbf{y}^{\prime} \mathbf{M} \mathbf{X} \mathbf{s}_{i}}{(T-1) \hat{\sigma}_{i i}}=\frac{\sum_{t=1}^{T}\left(y_{t}-\bar{y}\right)\left(x_{i t}-\bar{x}_{i}\right)}{\sum_{t=1}^{T}\left(x_{i t}-\bar{x}_{i}\right)^{2}}
\end{aligned}
$$

which is the same as the OLS estimate of the coefficient of $x_{i t}$ in the simple regression of $y_{t}$ on an intercept and $x_{i t}$ :

$$
y_{t}=\beta_{0}+\lambda_{i} x_{i t}+v_{i t}, \quad v_{i t} \backsim \operatorname{iid}\left(\mathbf{0}, \omega_{i}^{2}\right) .
$$

Again $\lambda_{i}$ can be of the opposite sign to $\beta_{i}{ }^{5}$

Although the overall impact effect, $\lambda_{i}$, can be estimated from simple regressions, when computing their standard errors (and the associated t-ratios) we need to take account of the multi-variate nature of the underlying regression model given by (17). To see this note that under (17) we have

$$
\mathbf{y}=\beta_{0} \boldsymbol{\tau}_{T}+\mathbf{X} \boldsymbol{\beta}+\mathbf{u},
$$

where $\boldsymbol{\tau}_{T}=(1,1, \ldots, 1)^{\prime}$, and $\mathbf{u}=\left(u_{1}, u_{2}, \ldots, u_{T}\right)^{\prime} \backsim i i d\left(\mathbf{0}, \omega^{2} \mathbf{I}_{T}\right)$, where $\mathbf{I}_{T}$ is an identity matrix of order $T$. Hence

$$
\begin{aligned}
\hat{\lambda}_{i} & =\frac{\mathbf{y}^{\prime} \mathbf{M} \mathbf{X} \mathbf{s}_{i}}{\sum_{t=1}^{T}\left(x_{i t}-\bar{x}_{i}\right)^{2}}=\frac{\mathbf{s}_{i}^{\prime} \mathbf{X}^{\prime} \mathbf{M}\left(\beta_{0} \boldsymbol{\tau}_{T}+\mathbf{X} \boldsymbol{\beta}+\mathbf{u}\right)}{\sum_{t=1}^{T}\left(x_{i t}-\bar{x}_{i}\right)^{2}}, \\
& =\frac{\mathbf{s}_{j}^{\prime} \mathbf{X}^{\prime} \mathbf{M} \mathbf{X} \boldsymbol{\beta}}{\sum_{t=1}^{T}\left(x_{i t}-\bar{x}_{i}\right)^{2}}+\frac{\mathbf{s}_{i}^{\prime} \mathbf{X}^{\prime} \mathbf{M u}}{\sum_{t=1}^{T}\left(x_{i t}-\bar{x}_{i}\right)^{2}},
\end{aligned}
$$

and under the above assumptions and for $T$ sufficiently large we have

$$
\hat{\lambda}_{i}-\lambda_{i} \stackrel{a}{\sim} N\left(0, \frac{\omega^{2}}{\sum_{t=1}^{T}\left(x_{i t}-\bar{x}_{i}\right)^{2}}\right),
$$

which has the same form as the asymptotic distribution of the OLS coefficient of $x_{j t}$ in the simple regression of $y_{t}$ on $x_{j t}$; but with the important difference that $\omega^{2}$ is the variance of the error term, $u_{t}$, in the multiple regression equation (17) and not the variance of the error in the simple regression (20). In fact we always have $\omega^{2} \leq \omega_{j}^{2}$ for all $j$. Therefore, when making inference about the sign of $\lambda_{j}$, we cannot rely on the t-ratio of $\lambda_{j}$ (say $t_{j}$ ) computed from (20), but we should adjust $t_{j}$ upward by the factor $\omega_{j} / \omega \geq 1$. Namely, the appropriate t-ratio to be used for inference about the sign of $\lambda_{j}$ is given by

$$
t_{j}^{*}=\left(\hat{\omega}_{j} / \hat{\omega}\right) t_{j} .
$$

\footnotetext{
${ }^{5}$ Leamer (1975) shows that the coefficient of the focus variable, $x_{2 t}$, will not change sign, when the control variable, $x_{1 t}$, is excluded if $\hat{\beta}_{2}$ is more significant than $\hat{\beta}_{1}$. McAleer, Pagan \& Visco (1986) provide necessary conditions for a change in sign of the focus variable if one drops a control variable. Kennedy (2005) gives a range of cases where one gets the "wrong" sign, some for this type of reason.
} 
where $\hat{\omega}_{j}$ and $\hat{\omega}$ are the unbiased estimators of $\omega_{j}$ and $\omega$ based on the simple and multiple regressions, (17) and (20), respectively. In some circumstances, one may not have data on all the possible regressors, but may have some view on the likely size of $\omega$ and thus may be able to put a bound on the adjustment.

When (17) is correctly specified and the full set of regressors is known the appropriate standard error can also be obtained by estimating for $j \neq i$ the residuals $\hat{u}_{j i, t}$ from

$$
x_{j t}=\hat{\gamma}_{i j, 0}+\hat{\gamma}_{j i} x_{i t}+\hat{u}_{j i, t}
$$

and estimating

$$
y_{t}=c+\lambda_{i} x_{i t}+\sum_{j=1, j \neq i}^{k} \beta_{j} \hat{u}_{j i, t}+u_{t},
$$

where $c$ is a constant. Since (22) is just a reparameterisation of (17), obtained by adding and subtracting $\left(\hat{\gamma}_{i j, 0}+\hat{\gamma}_{j i} x_{i t}\right)$, the least squares estimate of $u_{t}$ from (22) is identical to the estimate from (17). Thus these residuals can be used to obtain an unbiased estimator of $\omega^{2}$ which can be used in (21) to obtain an estimate of the standard error of $\hat{\lambda}_{i}$. This procedure of orthogonalising control variables with respect to the focus variable has been widely used in a number of disciplines, though rarely in econometrics, and has been equally widely criticised

because the least squares estimate $\hat{\lambda}_{i}$ gives a biased and inconsistent estimate of $\beta_{i}$, e.g. Freckleton (2002). This criticism is correct if the parameter of interest is $\beta_{i}$, but is irrelevant if the parameter of interest is $\lambda_{i}$ as argued above.

\section{Conclusion}

In this paper we have argued that in multiple time series regressions the regression coefficients do not necessarily measure the parameter of interest because the ceteris paribus assumption that underlies the controlled experimental set up is not appropriate. In many cases the impact effect of interest is the total effect, allowing the other regressors to adjust as one would expect them to from the historical correlations. In linear models this gives the same estimate as would be obtained by excluding the other control variables. The argument here is similar to that made by Pesaran and Smith (2012) who suggest that if $x_{i t}$ is a policy variable and $x_{j t}, j \neq i$, are other variables that are potentially influenced by the policy variable, then $x_{j t}$ should be excluded from the equation used to estimate the counterfactual, i.e. that $\lambda_{i}$ above in (20) or (22) is the relevant parameter estimate to use in constructing the counterfactual. For instance they consider quantitative easing (QE) in the UK, where it is assumed that QE changes the spread between long and short rates, $x_{i t}$, which influences other variables like the exchange rate, $x_{j t}$, while both the spread and these other variables influence growth, $y_{t}$. In this case these other variables should be excluded when estimating the effects of QE. 


\section{References}

Bergstrom, A Rex (1984) Continuous time stochastic models and issues of aggregation over time in Z. Grilliches and M.D. Intriligator (eds) Handbook of Econometrics, North Holland.

Freckleton, Robert P (2002) On the misuse of residuals in ecology: regression of residuals vs. multiple regression, Journal of Animal Ecology, 71(3), 542-545.

Kennedy, Peter E (2005) Oh No! I got the wrong sign! What should I do? The Journal of Economic Education, 36, 77-92.

Koop, Gary, M Hashem Pesaran and Simon Potter (1996), Impulse response analysis in nonlinear multivariate models, Journal of Econometrics, 74, 119-47.

Leamer, Edward (1975) A result on the sign of restricted least squares estimates, Journal of Econometrics, 3, 387-90.

McAleer, Michael, Adrian Pagan and Ignazio Visco (1986) A further result on the sign of restricted least squares estimate, Journal of Econometrics 32, 287-90.

Pesaran, M Hashem and Yongcheol Shin (1998) Generalised Impulse Response Analysis in Linear Multivariate Models. Economics Letters, 58, 17-29.

Pesaran, M Hashem and Ron P Smith (2012) Counterfactual Analysis in Macroeconometrics: An empirical investigation into the effects of quantitative easing, IZA Discussion Paper 6618. 